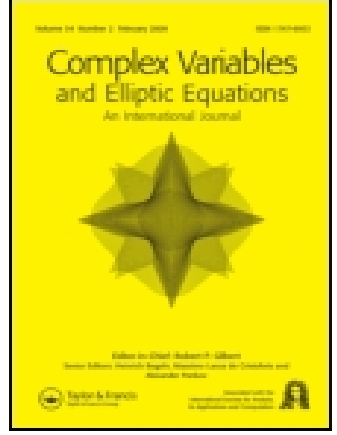

Complex Variables, Theory and Application: An International Journal

\title{
Parametric representation of a clas of multiply positive sequences
}

\section{I.V. Ostrovskii \& N.A. Zheltukhina}

To cite this article: I.V. Ostrovskii \& N.A. Zheltukhina (1998) Parametric representation of a clas of multiply positive sequences, Complex Variables, Theory and Application: An International Journal, 37:1-4, 457-469, DOI: 10.1080/17476939808815144

To link to this article: https://doi.org/10.1080/17476939808815144

曲 Published online: 29 May 2007.

Submit your article to this journal $\pi$

Џ Article views: 43

Citing articles: 2 View citing articles $\square$ 


\title{
Parametric Representation of a Class of Multiply Positive Sequences
}

\author{
I. V. OSTROVSKIII,b,* and N. A. ZHELTUKHINA ${ }^{b, \dagger}$
}

a Department of Mathematics, Bilkent University, 06533 Bilkent, Ankara, Turkey; ${ }^{\circ} B$. Verkin Institute for Low Temperature Physics and Engineering, 310164 Kharkov, Ukraine

Communicated by A. Eremenko

Dedicated to Professor A. A. Gol'dberg

(Received)

Keywords: Generating function; sequences; sequences of matrices

Classification Categories: 30D99

\section{INTRODUCTION}

Multiply positive sequences were introduced by Fekete [1] in 1912 for study of zeros of real polynomials and entire functions. Since then these sequences have been studied by several mathematicians and have found several applications in Analysis (see, e.g. [2,3]).

Recall that the sequence

$$
\left\{a_{n}\right\}_{n=0}^{\infty}, \quad a_{0}>0, \quad a_{n} \geq 0(n=1,2, \ldots),
$$

*E-mails: iossif@fen.bilkent.edu.tr, ostrovskii@ilt.kharkov.ua

†E-mail: zheltukhina@ilt.kharkov.ua 
is said to be $m$-times positive for $m \in N \cup\{\infty\}$, if all minors of orders < $m+1$ of the infinite matrix

$$
A=\left(\begin{array}{ccccc}
a_{0} & a_{1} & a_{2} & a_{3} & \ldots \\
0 & a_{0} & a_{1} & a_{2} & \ldots \\
0 & 0 & a_{0} & a_{1} & \ldots \\
0 & 0 & 0 & a_{0} & \ldots \\
. & . & . & . & \ldots
\end{array}\right)
$$

are non-negative. The class of all $m$-times positive sequences is denoted by $P F_{m}$.

Evidently, the class $P F_{1}$ consists of all sequences (1.1). The class $P F_{2}$ consists of all sequences of the form

$$
a_{n}=\exp \{-\psi(n)\}, \quad n=0,1,2, \ldots,
$$

where $\psi: N \cup\{0\} \rightarrow(-\infty,+\infty], \psi(0)<\infty$, is a convex function. In 1953, in joint works by Aissen, Edrei, Schoenberg, Whitney (see [2], p. 412) the complete solution of the problem of description of the class $P F_{\infty}$ was found:

THEOREM AESW. The function $f(z)=\sum_{n=0}^{\infty} a_{n} z^{n}$ is a generating function of a sequence of $P F_{\infty}$ if and only if

$$
f(z)=a_{0} \exp \{\gamma z) \frac{\prod_{i=1}^{\infty}\left(1+p_{i} z\right)}{\prod_{i=1}^{\infty}\left(1-q_{i} z\right)}
$$

where

$$
\begin{gathered}
a_{0}>0, \quad \gamma \geq 0, \quad p_{i} \geq 0, \quad q_{i} \geq 0 \quad(i=1,2,3, \ldots), \\
\sum_{i=1}^{\infty}\left(p_{i}+q_{i}\right)<\infty .
\end{gathered}
$$

Note that (1.3) gives the description of $P F_{\infty}$ in terms of independent parameters $a_{0}, \gamma, p_{i}, q_{i}(i=1,2,3, \ldots)$. This means: (i) arbitrary values of the parameters can be chosen under the only conditions (1.4); (ii) there is a one-to-one correspondance between collections of these parameters and sequences of $P F_{\infty}$. 
To the best of our knowledges, the problem of the description of the classes $P F_{m}, 3 \leq m<\infty$, in terms of independent parameters has not been solved until now. It is not clear even which kind of parameters could play the corresponding role (similar to that of $a_{0}, p_{i}, q_{i}$ and $\gamma$ in Theorem AESW).

This paper is devoted to description of a subclass $Q_{3} \subset P F_{3}$ in terms of independent parameters. The subclass was for the first time considered by the authors [5] in connection with a generalization of Pólya's theorem on sections of power series.

DEFINITION We say that a sequence (1.1) of $P F_{3}$ belongs to $Q_{3}$ if all truncated sequences $\left\{a_{n}\right\}_{n=0}^{k}=\left\{a_{0}, a_{1}, a_{2}, \ldots, a_{k}, 0,0, \ldots\right\}, k \in N$, belong to $P F_{3}$. (Note that in [5] we denoted by $Q_{3}$ the class of all corresponding generating functions.)

It turns out that the role of independent parameters describing $Q_{3}$ play points of the set

$$
(0, \infty) \times[0, \infty) \times \mathcal{U},
$$

where $\mathcal{U}$ is the set of all sequences $\left\{\alpha_{k}\right\}_{k=2}^{\infty}, 0 \leq \alpha_{k} \leq 1, k=2,3, \ldots$, such that if $\alpha_{j}=0$ for some $j$, then $\alpha_{k}=0$ for any $k \geq j$. To give a precise statement of our result, we define the numbers

$$
\begin{gathered}
{\left[\alpha_{2}\right]=1+\alpha_{2}, \quad\left[\alpha_{2} \alpha_{3}\right]=1+\alpha_{3} \sqrt{\left[\alpha_{2}\right]},} \\
{\left[\alpha_{2} \alpha_{3} \alpha_{4}\right]=1+\alpha_{4} \sqrt{\left[\alpha_{2} \alpha_{3}\right]}, \cdots} \\
{\left[\alpha_{2} \alpha_{3} \cdots \alpha_{n}\right]=1+\alpha_{n} \sqrt{\left[\alpha_{2} \alpha_{3} \cdots \alpha_{n-1}\right]}, \cdots}
\end{gathered}
$$

Our main result is the following theorem.

THEOREM 1. A sequence (1.1) belongs to $Q_{3}$ if and only if

$a_{1}=a_{0} \alpha, \quad a_{n}=\frac{a_{0} \alpha^{n} \alpha_{2}^{n-1} \alpha_{3}^{n-2} \cdots \alpha_{n-1}^{2} \alpha_{n}}{\left[\alpha_{2}\right]^{n / 2}\left[\alpha_{2} \alpha_{3}\right]^{(n-1) / 2} \cdots\left[\alpha_{2} \alpha_{3} \cdots \alpha_{n-1}\right]^{3 / 2}\left[\alpha_{2} \alpha_{3} \cdots \alpha_{n}\right]}$,

where

$$
a_{0}>0, \quad \alpha \geq 0, \quad\left\{\alpha_{n}\right\}_{n=2}^{\infty} \in \mathcal{U} .
$$

Thus, the independent parameters are $a_{0}, \alpha, \alpha_{2}, \alpha_{3}, \ldots$ The only restrictions on them are (1.8) i.e. belonging of the point $\left(a_{0}, \alpha, \alpha_{2}, \ldots\right)$ to the set (1.5). 
Since $Q_{3}$ is a subclass of $P F_{2}$, any sequence $\left\{a_{k}\right\}_{k=2}^{\infty}$ of $Q_{3}$ admits the representation (1.2). The question arises what additional conditions on the convex function $\psi$ imply that $\left\{a_{k}\right\}_{k=0}^{\infty}$ belongs to $Q_{3}$.

Denote

$$
N=\min \{n: \psi(n)=+\infty\} \quad(N=+\infty \text { if } \psi(n)<+\infty, \forall n \in N) .
$$

Set

$$
\Delta_{2} \psi(n):= \begin{cases}\psi(n)-2 \psi(n-1)+\psi(n-2), & 2 \leq n<N \\ +\infty, & n \geq N \text { (if } N<+\infty) .\end{cases}
$$

Define a sequence $\left\{w_{n}\right\}_{n=2}^{\infty}$, putting

$$
w_{2}=1, w_{n}=\left[\alpha_{2} \alpha_{3} \cdots, \alpha_{n-1}\right]_{\alpha_{2}=\alpha_{3}=\cdots=\alpha_{n-1}=1}, n \geq 3 .
$$

Evidently, the sequence $\left\{w_{n}\right\}_{n=2}^{\infty}$ is increasing and, for any $\left\{\alpha_{n}\right\}_{n=2}^{\infty} \in \mathcal{U}$, we have

$$
\begin{gathered}
1 \leq\left[\alpha_{2} \alpha_{3} \cdots \alpha_{n-1}\right] \leq w_{n}<\lim _{n \rightarrow \infty} w_{n}=1+c \\
c:=\frac{1+\sqrt{5}}{2}=1.613 \cdots
\end{gathered}
$$

THEOREM 2. For a sequence (1.2) to belong to $Q_{3}$ it is necessary and sufficient that

$$
\Delta_{2}(n) \geq \log \left(1+\frac{1}{\sqrt{w_{n}}}\right), \quad n \geq 2 .
$$

The equality holds in (1.11) for every $n \geq 2$ for the sequence (1.2) given by (1.7) with $\alpha_{2}=\alpha_{3}=\cdots=1$.

Evidently, the bound in the right hand side of (1.11) has the following properties: (i) it is contained in the half-closed interval $(\log c, \log 2]$, (ii) it is equal to $\log 2$ for $n=2$, (iii) it tends to $\log c$ as $n \rightarrow \infty$. Therefore, using the second assertion of Theorem 2 , we obtain the following corollary.

COROLLARY 1. For a sequence (1.2) to belong to $Q_{3}$ it is necessary to have

$$
\Delta_{2} \psi(n)>\log c, \quad n \geq 2,
$$


and it is sufficient to have

$$
\Delta_{2} \psi(n) \geq \log 2, \quad n \geq 2
$$

Both above conditions are unimprovable in the following sense: the first one ceases to be necessary if one replaces $\log c$ by a larger constant; the second one ceases to be sufficient if one replaces $\log 2$ by a smaller constant.

Note the following immediate corollary of Theorem 2.

COROLlARY 2. Let $\psi:[0,+\infty) \rightarrow(-\infty,+\infty)$ be a convex function of $C^{2}[0, \infty)$.

(i) If for all sufficiently large $x$ we have $\psi^{\prime \prime}(x)<\log c$, then the sequence (1.2) does not belong to $Q_{3}$.

(ii) If for all $x \geq 0$ we have $\psi^{\prime \prime}(x) \geq \log 2$, then the sequence (1.2) belongs to $Q_{3}$.

Theorem 2 and Corollaries 1,2 can be used to determine whether a given sequence belongs to $Q_{3}$.

Example Consider the sequence

$$
A(\beta, d):=\left\{\exp \left(-d n^{\beta}\right)\right\}_{n=0}^{\infty}, \quad \beta>0, d>0
$$

If $\beta \geq 2$, then the function $\psi(x):=d x^{\beta}$ satisfies $\Delta_{2} \psi(n) \geq \Delta_{2} \psi(2)=$ $d\left(2^{\beta}-2\right)$ for $n \geq 2$. By Corollary 1, (1.12), we have $A(\beta, d) \in Q_{3}$ for $d \geq(\log 2) /\left(2^{\beta}-2\right)$. By Theorem 2 , (1.11) with $n=2$, we have $A(\beta, d) \notin Q_{3}$ for $d<(\log 2) /\left(2^{\beta}-2\right)$. If $\beta<2$, then $\psi^{\prime \prime}(x) \rightarrow 0$, as $x \rightarrow \infty$. By Corollary 2 we have $A(\beta, d) \notin Q_{3}$.

Theorem 2 allows us to describe the possible character of the growth of $\psi$ for sequences (1.2) belonging to $Q_{3}$.

Define the lower order $\lambda[\psi]$ and the order $\rho[\psi]$ of $\psi$ as

$$
\lambda[\psi]=\liminf _{n \rightarrow \infty} \frac{\log \psi(n)}{\log n}, \quad \rho[\psi]=\limsup _{n \rightarrow \infty} \frac{\log \psi(n)}{\log n}
$$

Evidently, $\lambda[\psi] \leq \rho[\psi]$. Since, by (1.12),

$$
\psi(n) \geq \frac{n^{2}}{2} \log c+O(n), \quad n \rightarrow \infty,
$$


we have $\lambda[\psi] \geq 2$. The question arises about description of the pairs $(\lambda[\psi] ; \rho[\psi])$, for which the sequence (1.2) belongs to $Q_{3}$. Using Theorem 2 , we prove that $2 \leq \lambda[\psi] \leq \rho[\psi] \leq \infty$ is the only restriction.

THEOREM 3. For any pair of numbers $(a, b), 2 \leq a \leq b \leq \infty$, there is a sequence (1.2) of $Q_{3}$ such that for the corresponding function $\psi$ we have $\lambda[\psi]=a, \rho[\psi]=b$.

\section{PROOF OF THEOREM 1}

Recall two lemmas and some notation from [5].

LEMMA 1. [5] Let (1.1) be a sequence of $P F_{2}$. If $N<\infty$, where $N$ is defined by (1.9), then $a_{n}=0$ for any $n \geq N$.

For sequences (1.1) belonging to $P F_{2}$, Lemma 1 allows us to introduce the numbers

$$
\rho_{n}=\frac{a_{n-1}}{a_{n}}, \quad 1 \leq n<N ; \quad \delta_{n}=\frac{\rho_{n}}{\rho_{n-1}}, \quad 1 \leq n<N .
$$

Evidently,

$$
a_{n}=\frac{a_{0}}{\prod_{j=1}^{n} \rho_{j}}
$$

and

$$
a_{n}=\frac{a_{0} \alpha^{n}}{\delta_{2}^{n-1} \delta_{3}^{n-2} \cdots \delta_{n}}, \quad 1 \leq n<N
$$

where $\alpha=\left(1 / \rho_{1}\right)=\left(a_{1} / a_{0}\right)$.

LEMMA 2. [5] Let $\left\{a_{k}\right\}_{k=0}^{n}=\left\{a_{0}, a_{1}, \ldots, a_{n}, 0,0, \ldots\right\}, a_{0}>0, a_{n}>0$, $n \geq 2$ be a sequence of $P F_{3}$. Then

1) for $n=2$, we have $\delta_{2} \geq 2$,

2) for $n \geq 3$, we have $\delta_{n}>1$ and

$$
\left(\delta_{n}-1\right)^{2} \geq 1-\frac{1}{\delta_{n-1}}
$$


We start the proof of Theorem 1.

(i) Let (1.1) be a sequence of $Q_{3}$. Let us show that the representation (1.7) is valid. By Lemma 2 the numbers

$$
y_{n}:=\frac{1}{\delta_{n}-1}, \quad 2 \leq n<N
$$

are well-defined and the following inequalities hold:

$$
\left\{\begin{array}{l}
y_{n}^{2} \leq y_{n-1}+1, \quad 3 \leq n<N \\
y_{2} \leq 1
\end{array}\right.
$$

Define the parameters

$$
\alpha:=\frac{a_{1}}{a_{0}}, \quad \alpha_{n}:= \begin{cases}y_{2}, & n=2 ; \\ \frac{y_{n}}{\sqrt{1+y_{n-1}}}, & 3 \leq n<N ; \\ 0, & n \geq N,\end{cases}
$$

which will take part in (1.7). From (2.3), (2.4) it follows $\left\{\alpha_{n}\right\}_{n=2}^{\infty} \in \mathcal{U}$. From (2.4) we obtain

$$
y_{n}=\alpha_{n} \sqrt{1+\alpha_{n-1} \sqrt{1+\cdots+\alpha_{3} \sqrt{1+\alpha_{2}}}}, \quad 2 \leq n<N .
$$

Hence, by (2.2),

$$
\delta_{n}=1+\frac{1}{\alpha_{n} \sqrt{1+\alpha_{n-1} \sqrt{1+\cdots+\alpha_{3} \sqrt{1+\alpha_{2}}}}}, \quad 2 \leq n<N
$$

Using (2.1), we have for $2 \leq n<N$

$$
\begin{aligned}
& a_{n}=\frac{a_{0} \alpha^{n}}{\left(1+\frac{1}{\alpha_{2}}\right)^{n-1}\left(1+\frac{1}{\alpha_{3} \sqrt{1+\alpha_{2}}}\right)^{n-2} \ldots} \\
& \left(1+\frac{1}{\alpha_{n} \sqrt{1+\alpha_{n-1} \sqrt{1+\cdots+\alpha_{3} \sqrt{1+\alpha_{2}}}}}\right) \\
& =\frac{a_{0} \alpha^{n} \alpha_{2}^{n-1} \alpha_{3}^{n-2} \cdots \alpha_{n}}{\left(1+\alpha_{2}\right)^{n / 2}\left(1+\alpha_{3} \sqrt{1+\alpha_{2}}\right)^{(n-1) / 2} \cdots}
\end{aligned}
$$

and $a_{n}=0$ for $n \geq N$. Taking into account notation (1.6), we obtain (1.7). 
(ii) Take any sequence $\left\{\alpha_{n}\right\}_{n=2}^{\infty} \in \mathcal{U}$ and any numbers $a_{0}>0, \alpha \geq 0$, and form the sequence $\left\{a_{k}\right\}_{k=0}^{\infty}$ according to (1.7). Let us prove that $\left\{a_{k}\right\}_{k=0}^{\infty} \in Q_{3}$.

Let $i:=\min \left\{n: \alpha_{n}=0\right\}$ ( $i:=\infty$ if $\alpha_{n} \neq 0, \forall n \geq 2$ ). Then we have $a_{n}>0$ for $0 \leq n<i$, and $a_{n}=0$ for $n \geq i$. Form the sequence

$$
y_{n}:= \begin{cases}\alpha_{n} \sqrt{1+y_{n-1}}, & 3 \leq n<i, \\ \alpha_{2}, & n=2 .\end{cases}
$$

Since $0 \leq \alpha_{n} \leq 1, n \geq 2$, the following inequalities hold:

$$
\left\{\begin{array}{l}
y_{n} \leq \sqrt{1+y_{n-1}}, \quad 3 \leq n<i \\
y_{2} \leq 1
\end{array}\right.
$$

Denote

$$
\begin{cases}\delta_{n}=1+1 / y_{n}, & 2 \leq n<i \\ \delta_{n}=\infty, & n \geq i\end{cases}
$$

Then we have

$$
a_{n}=\frac{a_{0} \alpha^{n}}{\delta_{2}^{n-1} \delta_{3}^{n-2} \cdots \delta_{n}}, \quad n \geq 2 .
$$

The proof of $\left\{a_{n}\right\}_{n=0}^{\infty} \in Q_{3}$ is very close to that of Lemma 5 of [5]. Nevertheless, we will present the proof for the reader's convenience.

As in [5], we use the following theorem of Schoenberg.

THEOREM ([4]) Let $\left\{b_{n}\right\}_{n=0}^{k}$ be a finite sequence of numbers. Consider $m$ matrices

$$
B_{n}=\left(\begin{array}{ccccccccc}
b_{0} & b_{1} & b_{2} & \cdots & b_{k} & 0 & 0 & \cdots & 0 \\
0 & b_{0} & b_{1} & \cdots & b_{k-1} & b_{k} & 0 & \cdots & 0 \\
0 & 0 & b_{0} & \cdots & b_{k-2} & b_{k-1} & b_{k} & \cdots & 0 \\
. & . & . & \cdots & . & . & . & \cdots & . \\
0 & 0 & 0 & \cdots & . & . & . & \cdots & b_{k}
\end{array}\right), n=1,2, \ldots, m
$$

where $B_{n}$ consists of $n$ rows and $k+n$ columns. Assume the following condition is satisfied for $n=1,2, \ldots, m$ : all $n \times n$-minors of $B_{n}$, consisting of consecutive columns are strictly positive. Then the sequence $\left\{b_{0}, b_{1}, \ldots\right.$, $\left.b_{k}, 0,0 \ldots\right\}$ is m-times positive.

Fix any $k, 2 \leq k<i$, and consider the sequence

$$
\left\{a_{0}, a_{1}, \ldots, a_{k-1}, a_{k}-\varepsilon, 0,0, \ldots\right\} \text {, }
$$


where $\varepsilon>0$ will be chosen sufficiently small later. Form three matrices

$$
\begin{aligned}
A_{1} & =\left(\begin{array}{lllllll}
a_{0} & a_{1} & \cdots & a_{k-1} & a_{k}-\varepsilon
\end{array}\right), \\
A_{2} & =\left(\begin{array}{ccccccc}
a_{0} & a_{1} & a_{2} & \cdots & a_{k-1} & a_{k}-\varepsilon & 0 \\
0 & a_{0} & a_{1} & \cdots & a_{k-2} & a_{k-1} & a_{k}-\varepsilon
\end{array}\right), \\
A_{3} & =\left(\begin{array}{cccccccc}
a_{0} & a_{1} & a_{2} & \cdots & a_{k-1} & a_{k}-\varepsilon & 0 & 0 \\
0 & a_{0} & a_{1} & \cdots & a_{k-2} & a_{k-1} & a_{k}-\varepsilon & 0 \\
0 & 0 & a_{0} & \cdots & a_{k-3} & a_{k-2} & a_{k-1} & a_{k}-\varepsilon
\end{array}\right) .
\end{aligned}
$$

All minors of $A_{1}$ are evidently positive for $0<\varepsilon<a_{k}$. Since

$$
\frac{a_{n}}{a_{n+1}}=\alpha \delta_{2} \delta_{3} \cdots \delta_{n+1}, \quad 1 \leq n<i-1, \quad \delta_{n}>1,
$$

we have

$$
\frac{a_{n-1}}{a_{n}}<\frac{a_{n}}{a_{n+1}}, \quad n=1,2, \ldots, k-2 ; \quad \frac{a_{k-2}}{a_{k-1}}<\frac{a_{k-1}}{a_{k}-\varepsilon} .
$$

for sufficiently small $\varepsilon>0$. Therefore all minors of $A_{2}$ are positive for such $\varepsilon$.

Further, consider the determinants

$$
N_{n}=\left|\begin{array}{ccc}
a_{n} & a_{n+1} & 0 \\
a_{n-1} & a_{n} & a_{n+1} \\
a_{n-2} & a_{n-1} & a_{n}
\end{array}\right|, \quad 2 \leq n<i .
$$

In virtue (2.9), (2.7) and (2.6), we have

$$
\begin{aligned}
N_{n} & =a_{n}^{3}+a_{n+1}^{2} a_{n-2}-2 a_{n+1} a_{n} a_{n-1} \\
& =a_{n+1}^{2} a_{n-2}\left(\delta_{n+1}^{2} \delta_{n}+1-2 \delta_{n+1} \delta_{n}\right) \\
& =a_{n+1}^{2} a_{n-2} \delta_{n}\left(\left(\delta_{n+1}-1\right)^{2}+\frac{1}{\delta_{n}}-1\right) \\
& =a_{n+1}^{2} a_{n-2} \delta_{n}\left(\frac{1}{y_{n+1}^{2}}-\frac{1}{y_{n}+1}\right) \geq 0,
\end{aligned}
$$

where $2 \leq n<i-1$. Moreover, (2.7) and (2.6) yield non-negativity of the minor

$$
N_{1}=\left|\begin{array}{ccc}
a_{1} & a_{2} & 0 \\
a_{0} & a_{1} & a_{2} \\
0 & a_{0} & a_{1}
\end{array}\right|=a_{0} a_{1} a_{2} \delta_{2}\left(1-\frac{2}{\delta_{2}}\right)=a_{0} a_{1} a_{2} \delta_{2}\left(1-y_{2}\right)
$$


Now, consider $3 \times 3$-minors of $A_{3}$ consisting of consecutive columns:

$$
\begin{gathered}
M_{0}=\left|\begin{array}{ccc}
a_{0} & a_{1} & a_{2} \\
0 & a_{0} & a_{1} \\
0 & 0 & a_{0}
\end{array}\right|, \quad M_{1}=\left|\begin{array}{ccc}
a_{1} & a_{2} & a_{3} \\
a_{0} & a_{1} & a_{2} \\
0 & a_{0} & a_{1}
\end{array}\right|, \\
M_{n}=\left|\begin{array}{ccc}
a_{n} & a_{n+1} & a_{n+2} \\
a_{n-1} & a_{n} & a_{n+1} \\
a_{n-2} & a_{n-1} & a_{n}
\end{array}\right|, \quad n=2,3, \ldots, k-3 ; \\
M_{k-2}(\varepsilon)=\left|\begin{array}{ccc}
a_{k-2} & a_{k-1} & a_{k}-\varepsilon \\
a_{k-3} & a_{k-2} & a_{k-1} \\
a_{k-4} & a_{k-3} & a_{k-2}
\end{array}\right|, \\
M_{k-1}(\varepsilon)=\left|\begin{array}{ccc}
a_{k-1} & a_{k}-\varepsilon & 0 \\
a_{k-2} & a_{k-1} & a_{k}-\varepsilon \\
a_{k-3} & a_{k-2} & a_{k-1}
\end{array}\right|, \\
M_{k}(\varepsilon)=\left|\begin{array}{ccc}
a_{k}-\varepsilon & 0 & 0 \\
a_{k-1} & a_{k}-\varepsilon & 0 \\
a_{k-2} & a_{k-1} & a_{k}-\varepsilon
\end{array}\right| .
\end{gathered}
$$

Positivity of $M_{0}$ and $M_{k}(\varepsilon)$ for $0<\varepsilon<a_{k}$ is evident. Since

$$
\begin{gathered}
M_{1}=N_{1}+a_{3}\left|\begin{array}{cc}
a_{0} & a_{1} \\
0 & a_{0}
\end{array}\right|, \\
M_{n}=N_{n}+a_{n+2}\left|\begin{array}{cc}
a_{n-1} & a_{n} \\
a_{n-2} & a_{n-1}
\end{array}\right|, \quad n=2,3, \ldots, k-3 ; \\
M_{k-2}(\varepsilon)=N_{k-2}+\left(a_{k}-\varepsilon\right)\left|\begin{array}{ll}
a_{k-3} & a_{k-2} \\
a_{k-4} & a_{k-3}
\end{array}\right|,
\end{gathered}
$$

we have $M_{n}>0, n=1,2, \ldots, k-3$, and $M_{k-2}(\varepsilon)>0$. Further, since

$$
\begin{gathered}
M_{k-1}(0)=N_{k-1} \geq 0, \\
M_{k-1}^{\prime}(0)=2 a_{k-1} a_{k-2}-2 a_{k} a_{k-3}=2 a_{k} a_{k-2}\left(\frac{a_{k-1}}{a_{k}}-\frac{a_{k-3}}{a_{k-2}}\right)>0,
\end{gathered}
$$

we have $M_{k-1}>0$ for sufficiently small $\varepsilon$.

Applying Schoenberg's theorem, we conclude that the sequence (2.8) belongs to $P F_{3}$ for sufficiently small $\varepsilon>0$. Taking a limit as $\varepsilon \rightarrow 0$, we obtain that the truncated sequence $\left\{a_{n}\right\}_{n=0}^{k}=\left\{a_{0}, a_{1}, \ldots, a_{k}, 0,0, \ldots\right\}$ is 3-times positive and hence $\left\{a_{n}\right\}_{n=0}^{\infty} \in Q_{3}$. 


\section{PROOF OF THEOREM 2}

Assume $\left\{a_{k}\right\}_{k=0}^{\infty} \in Q_{3}$. Then the representation (1.7) of Theorem 1 yields the following expression of the function $\psi$ connected with $\left\{a_{k}\right\}_{k=0}^{\infty}$ by (1.2):

$$
\begin{aligned}
\psi(n)= & -\log \frac{a_{0} \alpha^{n} \alpha_{2}^{n-1} \alpha_{3}^{n-2} \cdots \alpha_{n}}{\left[\alpha_{2}\right]^{n / 2}\left[\alpha_{2} \alpha_{3}\right]^{(n-1) / 2} \cdots\left[\alpha_{2} \alpha_{3} \cdots \alpha_{n-1}\right]^{3 / 2}\left[\alpha_{2} \alpha_{3} \cdots \alpha_{n}\right]} \\
= & -\log \frac{a_{0} \alpha^{n}}{\left(1+\frac{1}{\alpha_{2}}\right)^{n-1}\left(1+\frac{1}{\alpha_{3} \sqrt{\left[\alpha_{2}\right]}}\right)^{n-2} \cdots} \\
= & -\log a_{0}-n \log \alpha+(n-1) \log \left(1+\frac{1}{\alpha_{n} \sqrt{\left[\alpha_{2} \alpha_{3} \cdots \alpha_{n-1}\right]}}\right) \\
& +(n-2) \log \left(1+\frac{1}{\alpha_{3} \sqrt{\left[\alpha_{2}\right]}}\right)+\cdots \\
& +\log \left(1+\frac{1}{\alpha_{n} \sqrt{\left[\alpha_{2} \alpha_{3} \cdots \alpha_{n-1}\right]}}\right) .
\end{aligned}
$$

This yields

$$
\begin{aligned}
& \Delta_{2} \psi(2)=\log \left(1+\frac{1}{\alpha_{2}}\right), \\
& \Delta_{2} \psi(n)=\log \left(1+\frac{1}{\alpha_{n} \sqrt{\left[\alpha_{2} \alpha_{3} \cdots \alpha_{n-1}\right]}}\right), \quad n \geq 3 .
\end{aligned}
$$

Using the definition of the numbers $w_{n}$ and (1.10), we conclude that

$$
\Delta_{2} \psi(n) \geq \log \left(1+\frac{1}{\alpha_{n} \sqrt{w_{n}}}\right) \geq \log \left(1+\frac{1}{\sqrt{w_{n}}}\right)
$$

i.e. $(1.11)$ is true.

Now, assume that (1.11) holds. Set

$$
a_{0}=\exp \{-\psi(0)\}, \quad a_{1}=\exp \{-\psi(1)\}, \quad \alpha=\exp \{\psi(1)-\psi(0)\}
$$

and show that the numbers $\alpha_{2}, \alpha_{3}, \ldots \in[0,1]$ can be chosen such that the representation (1.7) is true. 
Since by (1.11) we have $\Delta_{2} \psi(2) \geq \log 2$, the number $\alpha_{2}$ defined by the first equality (3.2) belongs to [0,1]. Further, we will define the numbers $\alpha_{3}, \alpha_{4}, \ldots$ inductively by means of the second equality (3.2). Since, for fixed $\alpha_{2}, \alpha_{3} \cdots \alpha_{n-1}(n \geq 3)$, the function $\log (1+$ $\left.\left(1 / \alpha_{n} \sqrt{\left[\alpha_{2} \alpha_{3} \cdots \alpha_{n-1}\right]}\right)\right)$ as a function of $\alpha_{n} \in[0,1]$ is decreasing and its range covers the closed half-ray $[\log 2, \infty]$, the number $\alpha_{n}$ is defined by (3.2) uniquely if $\alpha_{2}, \alpha_{3}, \ldots \alpha_{n-1}$ have been defined. Having defined $\left\{\alpha_{k}\right\}_{k=2}^{\infty} \in \mathcal{U}$, we have (3.2) and (3.1) for all $n \geq 2$. Inverting the calculations in (3.1), we see that the representation (1.7) holds. By Theorem 1, we conclude that $\left\{a_{k}\right\}_{k=0}^{\infty} \in Q_{3}$.

\section{PROOF OF THEOREM 3}

For $a=b=\infty$, it suffices to consider any sequence (1.8) with $\alpha_{n}=0$, $n \geq N$, where $N$ is any integer $\geq 1$. For such a sequence, we have $\psi(n)=+\infty$ for $n \geq N$.

Now, let us assume $2 \leq a \leq b<\infty$. Consider an auxiliary function

$$
\begin{aligned}
\psi_{0}(x) & =\exp \{\vartheta(x)\}, \\
\vartheta(x) & =\frac{1}{2}\{a+b+(b-a) \sin (\log \log \log x)\} \log x,
\end{aligned}
$$

well-defined for $x>x_{0}=e^{e}$. Evidently, $\vartheta(x) \geq a \log x \geq 2 \log x$ for $x>x_{0}$, therefore $\psi_{0}(x) \geq x^{2}, x>x_{0}$. Moreover,

$$
\lambda\left[\psi_{0}\right]=\liminf _{x \rightarrow \infty} \frac{\vartheta(x)}{\log x}=a, \quad \rho\left[\psi_{0}\right]=\limsup _{x \rightarrow \infty} \frac{\vartheta(x)}{\log x}=b .
$$

Since

$$
\begin{aligned}
& \vartheta^{\prime}(x)=\frac{a+b+(b-a) \sin (\log \log \log x)}{2 x}+o\left(\frac{1}{x}\right), \quad x \rightarrow+\infty, \\
& \vartheta^{\prime \prime}(x)=-\frac{a+b+(b-a) \sin (\log \log \log x)}{2 x^{2}}+o\left(\frac{1}{x^{2}}\right), \quad x \rightarrow+\infty,
\end{aligned}
$$

we have

$$
\begin{aligned}
& \psi_{0}^{\prime}(x)=\vartheta^{\prime}(x) \psi_{0}(x) \geq\left(\frac{a}{x}+o\left(\frac{1}{x}\right)\right) \psi_{0}(x), \quad x \rightarrow+\infty, \\
& \psi_{0}^{\prime \prime}(x)=\left\{\vartheta^{\prime \prime}(x)+\vartheta^{\prime 2}(x)\right\} \psi_{0}(x)
\end{aligned}
$$




$$
\begin{aligned}
= & \frac{1}{x^{2}}\left\{\frac{a+b+(b-a) \sin (\log \log \log x)}{2}\right. \\
& \left.\times\left(\frac{a+b+(b-a) \cdot \sin (\log \log \log x)}{2}-1\right)+o(1)\right\} \psi_{0}(x) \\
\geq & \frac{1}{x^{2}}\{a(a-1)+o(1)\} \psi_{0}(x) .
\end{aligned}
$$

Using the estimate $\psi_{0} \geq x^{2}, x>x_{0}$, we obtain

$$
\psi_{0}^{\prime \prime}(x) \geq a(a-1)+o(1) \geq 2+o(1) \geq 1
$$

for sufficiently large $x$.

Choose a constant $A$ so large that the function $\psi(x):=\psi_{0}(x+A)$ satisfies the condition $\psi^{\prime \prime}(x) \geq 1$ for all $x \geq 0$. Hence,

$$
\Delta_{2} \psi(n)=\int_{n-1}^{n} d x \int_{x-1}^{x} \psi^{\prime \prime}(u) d u \geq 1>\log 2 .
$$

Corollary 1 shows that the sequence $a_{k}=\exp \{-\psi(k)\}$ belongs to $Q_{3}$. Evidently,

$$
\lambda[\psi]=\lambda\left[\psi_{0}\right]=a, \quad \rho[\psi]=\rho\left[\psi_{0}\right]=b .
$$

The case $2 \leq a<b=+\infty$ can be considered analogously by choosing

$$
\begin{aligned}
\psi_{0}(x) & =\exp \{\vartheta(x)\} \\
\vartheta(x) & =\left\{a+(\log \log \log x) \sin ^{2}(\log \log \log x)\right\} \log x
\end{aligned}
$$

\section{References}

[1] Fekete, M. and Pólya, G. (1912). Über ein Problem von Laguerre. Rendiconti Circ. Math. Palermo., 34, 89-120.

[2] Karlin, S. (1968). Total Positivity, Stanford University Press, Stanford.

[3] Baker, G. A. and Graves-Morris, P. (1981). Padé Approximants, Addison-Wesley, London.

[4] Schoenberg, I. J. (1955). On the zeros of generating functions of multiply positive sequences and functions. Ann. Math., 62, 447-471.

[5] Ostrovskii, I. V. and Zheltukhina, N. A. On power series having sections with multiply positive coefficients and a theorem of Pólya. J. Lond. Math. Soc., (to appear). 